\title{
One-carbon metabolism, cognitive impairment and CSF measures of Alzheimer pathology: homocysteine and beyond
}

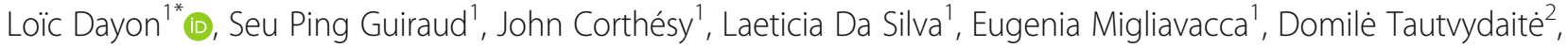
Aikaterini Oikonomidi ${ }^{2}$, Barbara Moullet ${ }^{2}$, Hugues Henry ${ }^{3}$, Sylviane Métairon ${ }^{1}$, Julien Marquis ${ }^{1}$, Patrick Descombes ${ }^{1}$, Sebastiano Collino', François-Pierre J. Martin ${ }^{1}$, Ivan Montoliu', Martin Kussmann ${ }^{1,5}$, Jérôme Wojcik ${ }^{4}$,

Gene L. Bowman ${ }^{1}$ and Julius Popp ${ }^{2}$

\begin{abstract}
Background: Hyperhomocysteinemia is a risk factor for cognitive decline and dementia, including Alzheimer disease (AD). Homocysteine (Hcy) is a sulfur-containing amino acid and metabolite of the methionine pathway. The interrelated methionine, purine, and thymidylate cycles constitute the one-carbon metabolism that plays a critical role in the synthesis of DNA, neurotransmitters, phospholipids, and myelin. In this study, we tested the hypothesis that one-carbon metabolites beyond Hcy are relevant to cognitive function and cerebrospinal fluid (CSF) measures of AD pathology in older adults.

Methods: Cross-sectional analysis was performed on matched CSF and plasma collected from 120 older community-dwelling adults with $(n=72)$ or without $(n=48)$ cognitive impairment. Liquid chromatography-mass spectrometry was performed to quantify one-carbon metabolites and their cofactors. Least absolute shrinkage and selection operator (LASSO) regression was initially applied to clinical and biomarker measures that generate the highest diagnostic accuracy of a priori-defined cognitive impairment (Clinical Dementia Rating-based) and AD pathology (i.e., CSF tau phosphorylated at threonine 181 [p-tau181]/B-Amyloid 1-42 peptide chain $\left[A \beta_{1-42}\right]>0.0779$ ) to establish a reference benchmark. Two other LASSO-determined models were generated that included the one-carbon metabolites in CSF and then plasma. Correlations of CSF and plasma one-carbon metabolites with CSF amyloid and tau were explored. LASSO-determined models were stratified by apolipoprotein E (APOE) $\varepsilon 4$ carrier status.

Results: The diagnostic accuracy of cognitive impairment for the reference model was $80.8 \%$ and included age, years of education, $A \beta_{1-42}$, tau, and p-tau181. A model including CSF cystathionine, methionine, S-adenosyl-L-homocysteine (SAH), S-adenosylmethionine (SAM), serine, cysteine, and 5-methyltetrahydrofolate (5-MTHF) improved the diagnostic accuracy to $87.4 \%$. A second model derived from plasma included cystathionine, glycine, methionine, SAH, SAM, serine, cysteine, and Hcy and reached a diagnostic accuracy of 87.5\%. CSF SAH and 5-MTHF were associated with CSF tau and p-tau181. Plasma one-carbon metabolites were able to diagnose subjects with a positive CSF profile of AD pathology in $A P O E$ \&4 carriers.

(Continued on next page)
\end{abstract}

\footnotetext{
* Correspondence: loic.dayon@rd.nestle.com

${ }^{1}$ Nestlé Institute of Health Sciences, École Polytechnique Fédérale de

Lausanne (EPFL) Innovation Park, Bâtiment H, 1015 Lausanne, Switzerland

Full list of author information is available at the end of the article
} 
(Continued from previous page)

Conclusions: We observed significant improvements in the prediction of cognitive impairment by adding one-carbon metabolites. This is partially explained by associations with CSF tau and p-tau181, suggesting a role for one-carbon metabolism in the aggregation of tau and neuronal injury. These metabolites may be particularly critical in $A P O E$ \&4 carriers.

Keywords: Alzheimer's disease, Cognition, CSF, Homocysteine, Metabolomics, One-carbon metabolism, S-adenosyl-L-homocysteine, Tau

\section{Background}

The methionine, purine, and thymidylate cycles together encompass one-carbon metabolism in the cytosol [1]. Both genetic (i.e., polymorphisms in MTHFR) and environmental factors influence one-carbon metabolism; however, genetic factors are thought to play a minor role, whereas dietary intake of folate, vitamin $B_{6}$, and vitamin $\mathrm{B}_{12}$ explains $35 \%$ of the variation [2].

The most-studied biomarker of one-carbon metabolism is homocysteine (Hcy), a sulfur-containing amino acid generated by the metabolism of methionine [3]. Inherited deficiencies in cystathionine synthase, methionine synthase, or methylene-tetrahydrofolate reductase enzymes causing homocystinuria and severe atherosclerotic plaques were discovered in children in the mid-20th century [4]. More recently, hyperhomocysteinemia has been identified as a risk factor for a multitude of conditions, including cognitive decline and Alzheimer dementia [5-10]. Elevated plasma $\mathrm{Hcy}$ is linked with deficiency in vitamins $\mathrm{B}_{6}$, $B_{12}$, and folate, as well as oxidative damage $[11,12]$. However, Hcy-lowering trials with B vitamins have met with mixed results that have dampened enthusiasm for targeting Hcy in the prevention of cognitive decline or Alzheimer disease (AD) progression [13]. There may be several explanations for this inconsistency, but one often overlooked is that emphasis on Hcy specifically may be insufficient in magnitude of effect under the constraints of current clinical trial designs. A role for other participants in the one-carbon cycle that are highly interactive with Hcy may have been underappreciated. For example, $S$ adenosylmethionine (SAM), $S$-adenosyl-L-homocysteine (SAH), and 5-methyltetrahydrofolate (5-MTHF) balance may operate on several mechanisms that support cognitive function, ranging from epigenetic modulation of synaptic function in the hippocampus to methylation reactions in the liver that convert phosphatidylethanolamine to phosphatidylcholine and facilitate the delivery of essential fatty acids (i.e., docosahexaenoic acid) to the plasma and the brain [14-18].

The $\varepsilon 4$ allele of apolipoprotein $\mathrm{E}(A P O E)$ gene is a well-known and major genetic risk factor for $A D$ [19]. Several recent studies have shown the interaction of $A P O E$ genotype and environmental factors, such as diet, on the risk of dementia and AD [20]. Nutrient intake effect on cognitive function might be influenced by $A P O E$ status, and the association between $\mathrm{B}_{12}$ and cognitive function was indeed shown to be stronger in $A P O E \& 4$ carriers [21]. The effects of vitamin $\mathrm{B}_{12}$ and Hcy on gray matter volume may also be influenced by $A P O E$ genotype in $\mathrm{AD}$ [22]. The clear interaction between Hcy, its cofactors, and $A P O E$ remains to be more broadly investigated.

Metabolomics is an approach with the potential to target and identify perturbations in specific pathways of interest by quantifying a wide range of biochemical compounds in tissue [23]. We leveraged this approach to test the hypothesis that comprehensive assessment of one-carbon metabolism in cerebrospinal fluid (CSF) and plasma would better explain cognitive impairment and CSF measures of AD pathology in older adults.

\section{Methods \\ Study population}

The participants with cognitive impairment were recruited among outpatients who were referred to the memory clinics, departments of psychiatry, and the Leenaards Memory Center, Department of Clinical Neurosciences, University Hospitals of Lausanne (Switzerland). Cognitively intact participants were recruited from the community through advertisement or among the spouses of memory clinic patients. In total, 120 subjects were enrolled in the biomarker study.

\section{CSF and plasma sample collection}

Venous and lumbar punctures were performed between 8:30 and 9:30 a.m. after overnight fasting. For lumbar puncture, a standardized technique with a 22-gauge "atraumatic" spinal needle and the subject in a sitting or lying position was applied [24]. A volume of 10-12 ml of CSF was collected in polypropylene tubes. CSF samples were centrifuged, frozen in aliquots, and stored at $-80{ }^{\circ} \mathrm{C}$ before further use. Blood was drawn into ethylenediaminetetraacetic acid-containing vacutainers. After 20-30 minutes on ice, the tubes were centrifuged. Plasma samples were aliquoted in polypropylene tubes and stored at $-80{ }^{\circ} \mathrm{C}$. 
CSF $\beta$-amyloid 1-42 peptide chain, tau, tau phosphorylated at threonine 181 , and $A P O E \varepsilon 4$ genotyping

The measurements were performed using commercially available enzyme-linked immunosorbent assay kits and TaqMan assays (Applied Biosystems, Foster City, CA, USA) as described in Additional file 1: Supplementary Methods.

\section{Targeted metabolomics of one-carbon metabolism}

Liquid chromatography-tandem mass spectrometry (LCMS/MS) was performed to measure absolute concentrations of metabolites and cofactors of one-carbon metabolism in CSF and blood plasma as described previously [23] (see Additional file 1: Supplementary Methods).

\section{Primary outcome measures Cognitive impairment}

Diagnosis of mild cognitive impairment (MCI) or dementia was based on neuropsychological and clinical evaluation and made by a consensus conference of psychiatrists and/or neurologists and neuropsychologists prior to subject inclusion. The participants had no major psychiatric or neurological disorders, nor did they have substance abuse or severe or unstable physical illness that might contribute to cognitive impairment. Magnetic resonance imaging and computed tomographic scans were used to exclude cerebral pathologies possibly interfering with the cognitive performance. Subjects known to take folate and B-vitamin supplementation were excluded from the study. MCI was diagnosed according to widely used consensus recommendations [25]. Subjects with MCI had memory impairment [26] and/or impairment in another cognitive domain such as executive tasks, as well as a Clinical Dementia Rating (CDR) [27] score of 0.5 . The diagnosis of probable $\mathrm{AD}$ dementia was defined according to the clinical diagnostic criteria for probable dementia due to $\mathrm{AD}$ according to the recommendations of the National Institute on Aging and the Alzheimer's Association [28] as well as the criteria of the Diagnostic and Statistical Manual of Mental Disorders, Fourth Edition, for dementia of the Alzheimer type [29] with a CDR of 1 . In the present study, we had only nine subjects with AD. Because there is a clinical continuum between $\mathrm{MCI}$ and mild dementia, and because the participants with cognitive impairment were patients from memory clinics recruited in the same way regardless of MCI or mild dementia classification, these subjects were collapsed with the MCI group and labeled as cognitively impaired. The participants without cognitive impairment had no history or evidence of cognitive deficits and required a CDR of 0 . We therefore defined two categories of subjects on the basis of their CDR (i.e., CDR 0 or $>0$ [i.e., 0.5 or 1 ]) (Table 1 ).

\section{CSF profiles of $A D$ pathology}

Subjects were also classified into two groups on the basis of their CSF tau phosphorylated at threonine 181 (p-tau181)/ $\beta$-amyloid $1-42$ peptide chain $\left(\mathrm{A} \beta_{1-42}\right)$ ratio: "low" when $\mathrm{p}$-tau181/A $\beta_{1-42}$ was $\leq 0.0779$ or "high" when $\mathrm{p}$-tau181/A $\beta_{1-42}$ was $>0.0779$, considered as negative and positive CSF profiles of AD pathology, respectively. This threshold optimized the Youden index [30] of the receiver operating characteristic (ROC) curve for the prediction of CDR categories (CDR 0 versus $C D R>0$ ) [31]. It was similar to previously reported findings [32]. Forty-two and 78 patients had positive and negative CSF profiles of AD pathology, respectively.

\section{Biomarker quality control}

Metabolites with $>5 \%$ missingness or below limits of quantification were excluded, which left eight CSF and plasma metabolites (Table 1). Data were $\log _{10}$-transformed to tend toward a Gaussian distribution and standardized to null average and SD of 1 prior to statistical analyses. One low-quality CSF sample was not analyzed. CSF and plasma metabolite data were available for 119 and 120 subjects, respectively.

\section{Statistical analyses}

Using least absolute shrinkage and selection operator (LASSO) logistic regression [33], we selected biomarkers predict both cognitive impairment and CSF profiles of AD pathology. A reference model was initially generated, testing variables that are likely to be available to the clinician and known risk factors for $\mathrm{AD}$ to provide a benchmark for comparison with the models that included one-carbon metabolites. These inputs included age, sex, years of education, presence of APOE $\varepsilon 4$ allele, CSF A $\beta_{1-42}$, CSF tau, and CSF p-tau181 concentrations for the prediction of cognitive impairment; and age, sex, years of education, and presence of $A P O E \varepsilon 4$ allele for the prediction of AD CSF profiles. In addition to all variables used to make the reference models, all metabolite measurements and CSF albumin index were then included in building so-called best models. The best models were built using LASSO regression by entering the CSF and plasma metabolites separately, thereby producing the two best models. A tenfold cross-validation process was performed for each LASSO analysis using the glmnet package in $\mathrm{R}$ [34], which allows estimating the confidence interval $(\mathrm{CI})$ of the misclassification error for each value of the regularization parameter $\lambda$. The LASSO analyses were repeated 100 times (1000 times for the reference model). The models that minimized the upper limit of the crossvalidated misclassification error $\mathrm{CI}$ across the 100 runs were selected. Their performance was assessed by ROC area under the curve (AUC) estimation using a bootstrap 
Table 1 Demographics and clinical characteristics

\begin{tabular}{|c|c|c|c|}
\hline & All $(n=120)$ & $\begin{array}{l}\text { Cognitively normal } \\
\text { (CDR 0) }(n=48)\end{array}$ & $\begin{array}{l}\text { Cognitively impaired } \\
\text { (CDR } 0.5 \text { or } 1)(n=72)\end{array}$ \\
\hline Age, years & $70.4(7.9)$ & $66.0(7.4)$ & $73.3(6.9)^{a}$ \\
\hline Male sex, $n(\%)$ & $43(35.83 \%)$ & $17(35.42 \%)$ & $26(36.11 \%)$ \\
\hline Education, years & $12.4(2.6)$ & $13.2(2.3)$ & $11.8(2.7)^{\mathrm{a}}$ \\
\hline MMSE score, mean & $26.9(3.1)$ & $28.5(1.4)$ & $25.9(3.5)^{\mathrm{a}}$ \\
\hline APOE $\varepsilon 4$ carrier, $n(\%)$ & 37 (30.83\%) & $11(22.92 \%)$ & $26(36.11 \%)^{a}$ \\
\hline \multicolumn{4}{|l|}{ CSF parameters } \\
\hline $\mathrm{A} \beta_{1-42}, \mathrm{pg} / \mathrm{ml}$ & $847.4(265.1)$ & $957.4(194.0)$ & $774.0(281.5)^{a}$ \\
\hline $\mathrm{tau}, \mathrm{pg} / \mathrm{ml}$ & $371.3(278.6)$ & $221.5(82.9)$ & $471.1(316.6)^{a}$ \\
\hline p-tau181, pg/ml & $62.0(35.2)$ & $45.9(13.3)$ & $72.7(40.9)^{\mathrm{a}}$ \\
\hline $\mathrm{p}$-tau181/A $\beta_{1-42}$ & $0.088(0.082)$ & $0.049(0.015)$ & $0.114(0.097)^{a}$ \\
\hline Albumin index ${ }^{b}$ & $6.1(2.4)$ & $5.3(1.9)$ & $6.6(2.5)^{\mathrm{a}}$ \\
\hline Choline, $\mu \mathrm{M}$ & $2.7(0.5)$ & $2.6(0.4)$ & $2.8(0.6)^{a}$ \\
\hline Cystathionine, nM & $35.8(17.0)$ & $34.4(13.8)$ & $36.7(18.9)$ \\
\hline Methionine, $\mu \mathrm{M}$ & $3.6(0.8)$ & $3.3(0.5)$ & $3.9(0.9)^{a}$ \\
\hline S-adenosylhomocysteine, nM & $15.1(5.8)$ & $13.5(4.3)$ & $16.1(6.3)^{a}$ \\
\hline S-adenosylmethionine, nM & $183.0(42.0)$ & $188.2(41.1)$ & $179.4(42.5)$ \\
\hline Serine, $\mu \mathrm{M}$ & $27.1(4.7)$ & $25.7(4.1)$ & $28.0(4.8)^{\mathrm{a}}$ \\
\hline Cysteine, $\mu \mathrm{M}$ & $1.2(0.4)$ & $1.2(0.4)$ & $1.3(0.4)$ \\
\hline 5-Methyltetrahydrofolate, nM & $45.1(12.5)$ & $47.2(10.9)$ & $43.8(13.4)$ \\
\hline \multicolumn{4}{|l|}{ Plasma parameters } \\
\hline Cystathionine, nM & $252.3(232.4)$ & $267.5(290.0)$ & $242.1(185.9)$ \\
\hline Glycine, $\mu \mathrm{M}$ & $234.5(60.6)$ & $235.9(59.7)$ & $233.6(61.5)$ \\
\hline Methionine, $\mu \mathrm{M}$ & $19.7(3.6)$ & $19.4(2.9)$ & $19.9(4.1)$ \\
\hline S-adenosylhomocysteine, nM & $20.9(9.1)$ & $18.3(6.3)$ & $22.7(10.2)^{a}$ \\
\hline S-adenosylmethionine, nM & $71.9(20.7)$ & $67.6(17.7)$ & $74.7(22.2)$ \\
\hline Serine, $\mu \mathrm{M}$ & $115.3(19.9)$ & $111.5(16.9)$ & $117.9(21.3)$ \\
\hline Cysteine, $\mu \mathrm{M}$ & $158.3(20.3)$ & $150.7(18.4)$ & $163.3(20.1)^{a}$ \\
\hline Homocysteine, $\mu \mathrm{M}$ & $5.6(1.6)$ & $5.3(1.5)$ & $5.8(1.7)$ \\
\hline
\end{tabular}

Abbreviations: A $\beta$-Amyloid, APOE Apolipoprotein E, CDR Clinical Dementia Rating, CSF Cerebrospinal fluid, MMSE Mini Mental State Examination, $p$-tau181 Tau phosphorylated at threonine 181

Data are given as means with SD within parentheses, unless indicated otherwise

${ }^{a}$ Statistically different $(p \leq 0.05)$ from CDR 0 using $t$ tests for continuous variables and binomial proportion tests for categorical variables

${ }^{b}$ CSF albumin index $=[$ CSF albumin $] /[$ serum albumin $] \times 100$

approach with 1000 iterations [35]. Results were compared visually and formally tested for significance against the reference model using ROC AUC [36] and accuracy using the McNemar test.

Two-sided correlation analyses between metabolites and CSF $A \beta_{1-42}$, tau, and p-tau181 were performed with Pearson's statistics and Bonferroni-corrected for multiple comparisons. Significant correlations between the metabolites and cofactors $(p<0.05)$ with CSF tau or $\mathrm{p}$ tau181 were studied further in a linear regression model with tau or p-tau181 as the dependent variable and the following explanatory covariates: each metabolite, age, sex, APOE genotype (presence versus absence of an APOE $\varepsilon 4$ allele), CDR ( 0 versus $>0$ ), and CSF albumin index (only for plasma metabolites). Values of the regression terms were reported, and their differences from 0 were assessed with $t$ tests. Interaction terms between the metabolites and age, sex, CDR, or APOE genotype were identified in a type II analysis of variance (ANOVA) with an $F$ test.

\section{Results}

Demographic and clinical characteristics of the study population

The cognitively impaired subjects were older and less educated and had a higher prevalence of the APOE $\varepsilon 4$ genotype than the cognitively intact group (CDR 0 ) (Table 1). CSF $A \beta_{1-42}$ was lower and CSF tau, CSF ptau181, CSF $\mathrm{p}$-tau181/A $\beta_{1-42}$, and CSF albumin index 
were all higher in those with cognitive impairment. Concentrations of the one-carbon metabolites in CSF and plasma are provided in Table 1.

\section{Reference benchmark for the prediction of cognitive impairment}

The reference model for classification of cognitive impairment included age, years of education, CSF $A \beta_{1-42}, C S F$ tau, and CSF p-tau181 and produced a diagnostic accuracy of $80.8 \%$ and an ROC AUC of 0.86 (95\% CI 0.79-0.92). This model classified 59 of 72 cognitively impaired subjects correctly and 38 of 48 cognitively intact subjects correctly. By comparison, a majority class prediction [37] produced a diagnostic accuracy of about $60 \%$ to distinguish subjects with CDR $>0$ from subjects with CDR 0 .

\section{CSF one-carbon metabolism and prediction of cognitive impairment}

After adding the CSF one-carbon metabolites, the diagnostic accuracy reached $87.4 \%$ (McNemar $p$ value of 0.0704 compared with reference model), and the ROC AUC reached 0.92 (CI 0.87-0.96) ( $p=0.0732$ versus the reference model, as shown in Fig. 1a). The CSF metabolites included cystathionine, methionine, SAH, SAM, serine, cysteine, and 5-MTHF. Age, years of education, CSF $A \beta_{1-42}$, CSF tau, CSF p-tau181, and CSF albumin index were also retained in the best model.

To evaluate the individual contribution of each metabolite selected in the best models, we conducted group comparisons for each metabolite. CSF methionine and serine were higher in cognitive impairment, with $p$ values of $5.5 \times 10^{-6}$ and $5.5 \times 10^{-3}$, respectively (Table 1 and Additional file 1: Figure S1). We also explored other CSF metabolites not chosen in the best model and observed CSF SAH $\left(p=8.8 \times 10^{-3}\right)$ and choline $(p=0.01)$ elevation in the cognitively impaired patients.

\section{Plasma one-carbon metabolism and prediction of cognitive impairment}

After adding the plasma one-carbon metabolites in the prediction of cognitive impairment, the diagnostic accuracy reached $87.5 \%$ (McNemar $p$ value of 0.0614 ), and the ROC AUC was improved to 0.91 (CI 0.86$0.96)(p=0.0028$ versus the reference model, as shown in Fig. 1b). The best model included plasma cystathionine, glycine, methionine, SAH, SAM, serine, cysteine, and Hcy. Age, sex, years of education, presence of APOE $\varepsilon 4$ allele, CSF $\mathrm{A} \beta_{1-42}$, CSF tau, CSF p-tau181, and CSF albumin index were in this best model. Plasma cysteine and SAH were elevated in cognitively impaired individuals, with $p$ values of $5.5 \times 10^{-4}$ and $4.6 \times 10^{-3}$, respectively (Table 1 and Additional file 1: Figure S2).

\section{Reference benchmark for prediction of CSF profile of AD pathology}

The reference model for classification of the CSF profile of $\mathrm{AD}$ pathology included age and presence of $A P O E \varepsilon 4$ allele. It had a diagnostic accuracy of $78.3 \%$ and an ROC AUC of 0.83 (CI 0.74-0.90). A majority class prediction produced a diagnostic accuracy of about $65 \%$ to distinguish subjects with $\mathrm{p}$-tau181/A $\beta_{1-42} \leq 0.0779$ from subjects with $\mathrm{p}$-tau181/A $\beta_{1-42}>0.0779$.
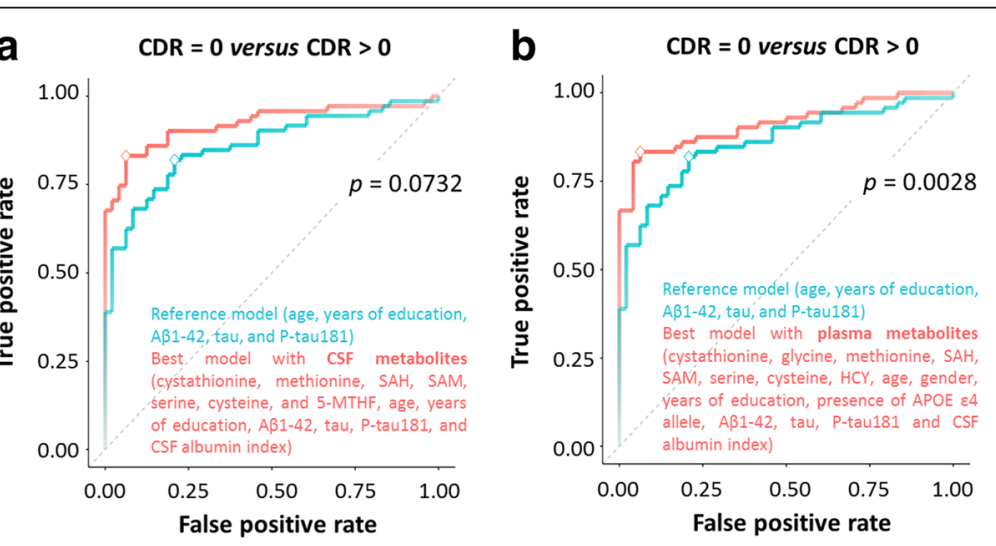

Fig. 1 ROC curves of the (a) cerebrospinal fluid (CSF) and (b) plasma models predictive of cognitive impairment (Clinical Dementia Rating [CDR] 0 or $>0$ ). The CSF model includes cystathionine, methionine, S-adenosyl-L-homocysteine (SAH), S-adenosylmethionine (SAM), serine, cysteine, and 5-methyltetrahydrofolate (5-MTHF) in addition to age, years of education, CSF $\beta$-amyloid 1-42 peptide chain (A $\beta_{1-42}$ ), CSF tau, CSF tau phosphorylated at threonine 181 (p-tau181), and CSF albumin index. The plasma model includes cystathionine, glycine, methionine, SAH, SAM, serine, cysteine, and homocysteine (Hcy) in addition to age, sex, years of education, presence of apolipoprotein E (APOE) $\varepsilon 4$ allele, CSF A $\beta_{1-42}$, CSF tau, CSF p-tau181, and CSF albumin index. The ROC curves of the least absolute shrinkage and selection operator (LASSO) models including metabolites (in red) are compared with the ROC curves of the reference LASSO models (in blue). The reference model is composed of age, years of education, CSF A $\beta_{1-42}$, CSF tau, and CSF p-tau181. The opacity of the curves is proportional to the accuracy of the models. The diamonds indicate the selected most accurate models. The $p$ values on the graphs indicate the significance of the differences of AUC. APOE Apolipoprotein E, HCY Homocysteine, 5-MTHF 5-Methyltetrahydrofolate, SAH S-adenosyl-L-homocysteine, SAM S-adenosylmethionine 
CSF and plasma one-carbon metabolism and prediction of CSF profile of AD pathology

CSF and plasma one-carbon metabolites were both unable to improve the classification of CSF profile of AD pathology beyond the reference model (data not shown).

\section{One-carbon metabolites and AD pathology}

CSF levels of $A \beta_{1-42}$, tau, and p-tau181 were significantly different between CDR categories (Table 1). The correlations of individual metabolite levels in CSF and plasma with those markers of $\mathrm{AD}$ pathology were therefore assessed. None of the one-carbon metabolites correlated with CSF A $\beta_{1-42}$. On the contrary, CSF cystathionine, SAH, and 5-MTHF as well as plasma Hcy were correlated with CSF p-tau181 (Fig. 2a). CSF SAH and 5MTHF were significantly associated with CSF tau (Fig. 2b). CSF 5-MTHF was inversely correlated with both CSF p-tau181 and tau (Fig. 2).

Metabolites significantly correlated with CSF p-tau181 or CSF tau were studied further in a linear regression model. The coefficients of each regression term and their significance are summarized in Table 2 for $p$ tau181 and tau. The cognitive impairment status term (CDR 0 versus $C D R>0$ ) was found to be associated in all models, confirming the association of high levels of CSF tau and CSF p-tau181 with cognitive impairment. Interaction terms between metabolite biomarkers and age, sex, CDR, and $A P O E \& 4$ genotype were then added to the regression models and tested by ANOVA (Table 3). An interaction was observed for CSF p-tau181 between plasma Hcy and APOE \&4 genotype (Fig. 3a). For CSF p-tau181, significant interactions were also obtained between SAH and 5-MTHF in CSF and APOE $\varepsilon 4$ genotype. Sex was found to interact with CSF SAH and plasma Hcy for CSF p-tau181 and with CSF SAH for CSF tau.

\section{Interaction of $A P O E$ \&4 genotype in one-carbon metabolite-based prediction of AD pathology}

Because we observed some metabolite interactions with $A P O E$ genotype, we stratified the analysis on the basis of individuals carrying the $A P O E \& 4$ allele $(n=37)$. In this subgroup, no correlation was found with CSF levels of $\mathrm{A} \beta_{1-42}$ and individual metabolite levels in both CSF and plasma. Cystathionine, SAH, and 5-MTHF in CSF, as well as Hcy in plasma, showed significant correlations with CSF p-tau181 (Additional file 1: Figure S3a). Concentrations of SAH and 5-MTHF in CSF and Hcy in plasma were significantly associated with CSF tau (Additional file 1: Figure S3b). Again, however, except for cognitive impairment status (CDR 0 versus $C D R>0$ ), these observed correlations were not impacted by other clinical covariates (data not shown).

The metabolite measurements were considered in a LASSO logistic regression to classify patients with non$A D$ versus $A D$ CSF profiles in this subgroup of individuals. A reference LASSO logistic regression on known or probable contributing variables (i.e., age and years of education) provided a reference model with an accuracy of $83.8 \%$ and an ROC AUC of 0.76 (CI 0.55-0.92). Here, a majority class classifier provided $64.9 \%$ diagnostic accuracy. Including metabolite markers measured in CSF did not improve the ability to predict an AD biomarker profile compared with the reference model. In contrast, plasma metabolites provided nonsignificant improvement in terms of accuracy but significantly increased performance for AUC (Fig. 3b). The model with plasma metabolites presented an accuracy of $89.2 \%$ (McNemar $p$ value of 0.6831 ) and an ROC AUC of 0.92 (CI 0.7$1.00)(p=0.0129$ versus the reference model). Seven
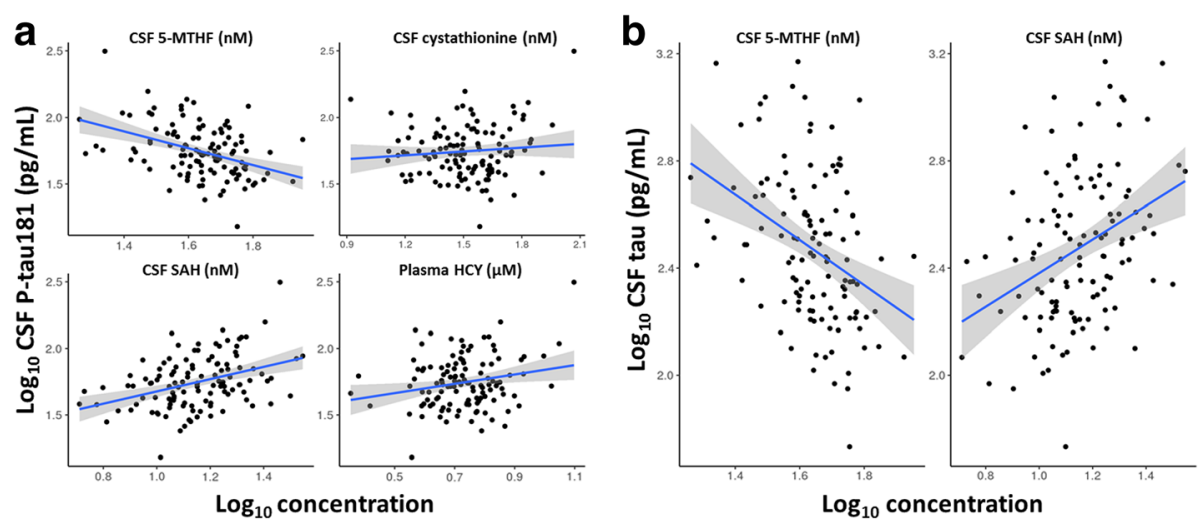

Fig. 2 Cerebrospinal fluid (CSF) and plasma metabolites correlated with CSF tau phosphorylated at threonine 181 (p-tau181) (a) and CSF tau (b). Concentration values were $\log _{10}$-transformed. Each dot represents a subject. The black line represents the linear fit, and the gray shading represents its confidence interval. HCY Homocysteine, 5-MTHF 5-Methyltetrahydrofolate, SAH S-adenosyl-L-homocysteine, SAM S-adenosylmethionine 
Table 2 Regression coefficients in linear model with cerebrospinal fluid tau phosphorylated at threonine 181 and tau as dependent variables

\begin{tabular}{|c|c|c|c|c|}
\hline Regression term & CSF cystathionine & CSF SAH & CSF 5-MTHF & Plasma Hcy \\
\hline \multicolumn{5}{|l|}{ CSF p-tau181 } \\
\hline Intercept & $1.0719^{\mathrm{a}}$ & $1.0503^{\mathrm{a}}$ & $2.1744^{\mathrm{a}}$ & $1.1802^{\mathrm{a}}$ \\
\hline Age & $0.0063^{b}$ & 0.0038 & $0.0045^{c}$ & $0.0054^{c}$ \\
\hline Sex $x^{d}$ & -0.0279 & -0.0223 & -0.0140 & -0.0236 \\
\hline$A P O E \varepsilon 4$ genotype & 0.0328 & 0.0195 & 0.0362 & 0.0314 \\
\hline CDR category ${ }^{f}$ & $0.1173^{b}$ & $0.1182^{b}$ & $0.1110^{\mathrm{b}}$ & $0.1186^{b}$ \\
\hline Biomarker & 0.1120 & $0.3195^{b}$ & $-0.4965^{a}$ & 0.1670 \\
\hline \multicolumn{5}{|l|}{ CSF tau } \\
\hline Intercept & & $1.4134^{\mathrm{a}}$ & $2.8174^{\mathrm{a}}$ & \\
\hline Age & & $0.0073^{c}$ & $0.0080^{c}$ & \\
\hline Sex ${ }^{d}$ & & -0.0215 & -0.0108 & \\
\hline APOE $\varepsilon 4$ genotype & & 0.0692 & 0.0894 & \\
\hline CDR category ${ }^{f}$ & & $0.1758^{\mathrm{a}}$ & $0.1665^{b}$ & \\
\hline Biomarker & & $0.3803^{b}$ & $-0.6269^{a}$ & \\
\hline
\end{tabular}

Abbreviations: A $\beta$ ß-Amyloid, APOE Apolipoprotein E, CDR Clinical Dementia Rating, CSF Cerebrospinal fluid, Hcy Homocysteine, 5-MTHF 5-Methyltetrahydrofolate, p-tau181 Tau phosphorylated at threonine 181, SAH S-adenosyl-L-homocysteine

Values of the regression terms are reported, and their differences from 0 were assessed with a $t$ test

${ }^{a} p \leq 0.001$

${ }^{\mathrm{b}} p \leq 0.01$

${ }^{c} p \leq 0.05$

${ }^{\mathrm{d}}$ Females (versus reference males)

${ }^{\mathrm{e}} A P O E \varepsilon 4$ carriers (versus reference noncarriers)

${ }^{f} \mathrm{CDR}>0$ (versus reference $\mathrm{CDR} 0$ )

Table 3 Regression coefficients in linear model with cerebrospinal fluid tau phosphorylated at threonine 181 and tau as dependent variables of interaction terms between biomarkers and different variables

\begin{tabular}{|c|c|c|c|c|}
\hline Interaction term & CSF cystathionine & CSF SAH & CSF 5-MTHF & Plasma Hcy \\
\hline \multicolumn{5}{|l|}{ CSF p-tau181 } \\
\hline Age & -0.0011 & -0.0098 & 0.0036 & 0.0113 \\
\hline $\operatorname{Sex}^{a}$ & -0.0652 & $-0.4815^{b}$ & 0.3443 & $-0.6850^{\mathrm{b}}$ \\
\hline$A P O E$ \&4 genotype ${ }^{c}$ & 0.2169 & $0.5126^{b}$ & $-0.5687^{b}$ & $1.1427^{d}$ \\
\hline CDR category ${ }^{e}$ & 0.1877 & 0.2122 & -0.2434 & 0.2833 \\
\hline \multicolumn{5}{|l|}{ CSF tau } \\
\hline Age & & -0.0076 & 0.0099 & \\
\hline $\operatorname{Sex}^{\mathrm{a}}$ & & $-0.7407^{f}$ & 0.4541 & \\
\hline$A P O E \varepsilon 4$ genotype ${ }^{c}$ & & 0.4953 & -0.6965 & \\
\hline CDR category $^{\mathrm{e}}$ & & 0.1352 & -0.2681 & \\
\hline
\end{tabular}

Abbreviations: APOE Apolipoprotein E, CDR Clinical Dementia Rating, CSF Cerebrospinal fluid, Hcy Homocysteine, 5-MTHF 5-Methyltetrahydrofolate, $p$-tau181 Tau phosphorylated at threonine 181, SAH S-adenosyl-L-homocysteine

Every interaction was tested in separate models, with only one interaction per model. Values of the regression interaction terms are reported, and their difference from 0 was assessed with an $F$ test in analysis of variance

${ }^{a}$ Females (versus reference males)

${ }^{\mathrm{b}} p \leq 0.05$

${ }^{\circ} A P O E \varepsilon 4$ carriers (versus reference noncarriers)

${ }^{d} p \leq 0.001$

${ }^{e} \mathrm{CDR}>0$ (versus reference CDR 0)

${ }^{\mathrm{f}} p \leq 0.01$ 


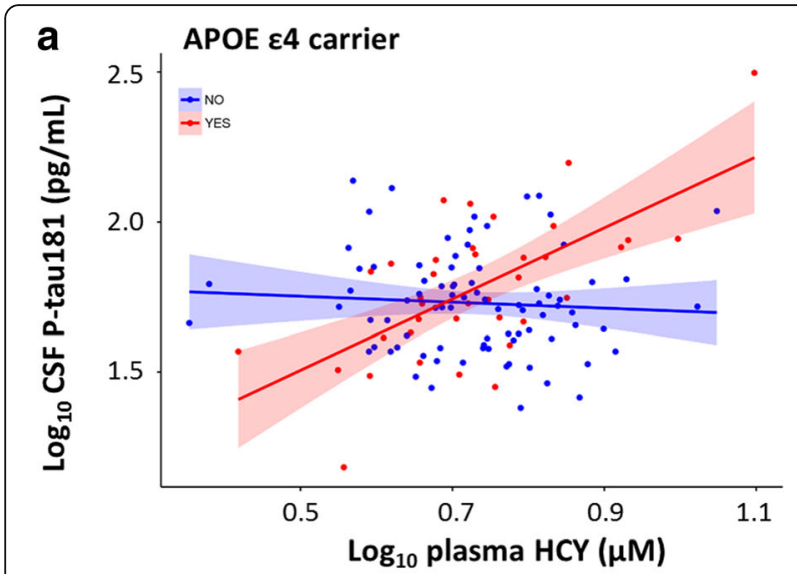

b Non-AD versus AD CSF profiles

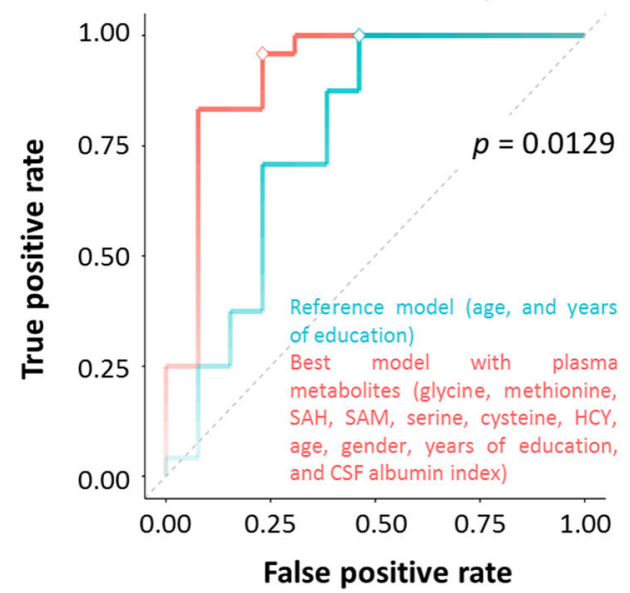

Fig. 3 Association of homocysteine (Hcy) levels measured in plasma with cerebrospinal fluid (CSF) tau phosphorylated at threonine 181 ( $p$-tau181), stratified by the presence of the apolipoprotein E (APOE) $\varepsilon 4$ allele (a). The correlation between expression levels of plasma Hcy and CSF p-tau181 differs according to APOE genotype. ROC curves of the models predictive of non-Alzheimer disease (non-AD) versus $A D$ CSF profiles (i.e., $p$-tau181/A $\beta_{1-42} \leq 0.0779$ and $p$-tau181/ $A \beta_{1-42}>0.0779$, respectively) (b). The ROC curves of the least absolute shrinkage and selection operator (LASSO) models including metabolites in plasma (in red) are compared with the ROC curves of the reference LASSO models (in blue). The plasma model (in red) includes glycine, methionine, S-adenosyl-L-homocysteine (SAH), S-adenosylmethionine (SAM), serine, cysteine, and Hcy in addition to age, sex, years of education, and CSF albumin index. The reference model (in blue) includes age and years of education. The opacity of the curves is proportional to the accuracy of the models. The diamonds indicate the selected most accurate models. The $p$ value on the graph indicates the significance of the differences of AUC. APOE Apolipoprotein E, HCY Homocysteine, SAH S-adenosyl-L-homocysteine, SAM S-adenosylmethionine

plasma metabolites were included in this mathematical model (i.e., glycine, methionine, SAH, SAM, serine, cysteine, and Hcy) in addition to age, sex, years of education, and CSF albumin index. Box plots of plasma metabolite measurements for non-AD versus AD CSF profiles in $A P O E$ $\varepsilon 4$ carriers are provided in Additional file 1: Figure S4.

\section{Discussion}

In the present study, we deployed a LC-MS/MS-based method to expand previous work [38], targeting onecarbon metabolism in both CSF and plasma, and to better understand its role in cognitive impairment and AD pathology. We identified CSF and plasma profiles including several one-carbon metabolites beyond Hcy associated with cognitive impairment in older adults. The association between several CSF and plasma one-carbon metabolites with CSF tau and p-tau181 warrants further research. Although we were unable to predict an a priori-stated CSF profile of AD pathology in the total cohort, the plasma one-carbon metabolites markedly improved the classification of CSF profiles of AD pathology in APOE $\varepsilon 4$ carriers.

Our results highlight the contribution of methionine, serine, choline, and cysteine in addition to the more commonly assessed and reported one-carbon metabolites (i.e., SAM, SAH, and Hcy). Methionine supplementation in wild-type mice can induce neurotoxicity, higher levels of tau phosphorylation and $A \beta$ peptides in the brain, and memory loss [39]. These mechanisms may explain our observation of higher CSF methionine in cognitive impairment. Protective effects of choline against age-related cognitive deficits were previously evidenced in animal models [40], and better memory performance was related to a higher concurrent choline intake in a large, nondemented, community-based human population [41]. However, we observed higher CSF choline in cognitive impairment, an inconsistency that may reflect neurodegeneration and the breakdown of synaptic membranes enriched with choline [42]. We showed significantly increased CSF and plasma SAH in cognitive impairment, which is consistent with previous findings $[14,43]$.

Several metabolites of the one-carbon cycle measured in CSF and plasma were associated with tau metabolism, reflected by total tau and p-tau181 in the CSF, after controlling for potential confounders. Higher CSF 5-MTHF appeared protective, whereas higher CSF SAH appeared to promote tau aggregation and neuronal injury. These results are consistent with reports demonstrating higher CSF SAH and lower CSF 5-MTHF associated with higher CSF p-tau181 [14]. Accordingly, impairment of the one-carbon metabolism, induced by feeding with a high-methionine/low-folate diet, was shown to increase SAH levels, downregulate methyltransferase, reduce protein phosphatase $2 \mathrm{~A}$ methylation, and induce accumulation of neurofibrillary tangle pathology in mice [44]. Higher levels of tau phosphorylation were also observed in the brains of wild-type mice fed a methionine-enriched diet, putatively inducing the fragmentation of tau from microtubules and leading to fibrillization [39]. Together, these results suggested that altered one-carbon 
metabolism may contribute to microtubule-associated tau protein hyperphosphorylation and neurodegeneration in general. Our inability to observe a relationship between any one-carbon metabolites with CSF $A \beta_{1-42}$ was remarkable and consistent with some studies [14, 45], but in contradiction with other in vitro and in vivo preclinical investigations $[46,47]$. A recent study of human adults with normal cognition found that disturbed onecarbon metabolism may be related to increased CSF levels of $A \beta_{1-42}$ and soluble amyloid precursor protein forms, suggesting a contribution to the accumulation of cerebral amyloid pathology [48], making it difficult to draw conclusions at this time.

We were unable to predict CSF profiles of AD pathology until an interaction with $A P O E$ genotype was identified. For example, whereas plasma Hcy appeared insubstantial to CSF tau in the total sample, hyperhomocysteinemia was relevant to CSF tau in the APOE $\varepsilon 4$ carriers. This was further corroborated by our prediction of positive AD pathology using the $\mathrm{p}$-tau181/A $\beta_{1-42}$ ratio after restricting the analysis to $A P O E \& 4$ carriers only. Although this interaction between Hcy, $A P O E$, and tau pathology was clear in our analysis, the biological rationale behind this interaction is less clear. A recent report demonstrated that plasma Hcy predicts the conversion from $\mathrm{MCI}$ to $\mathrm{AD}$ only in $A P O E$ \&4 carriers [49], adding a further level of consistency to our findings. Other metabolites of the one-carbon cycle, such as CSF SAM [50], may also be more relevant in $A P O E \varepsilon 4$ carriers and should be further explored.

Taken together, our results show that one-carbon metabolism is relevant to cognition independent of and beyond AD core pathology. One-carbon metabolism disturbances may cripple DNA repair, methylation, and/or synthesis, as well as reduce the availability of neurotransmitters, phospholipids, and myelin [51]. Several of these mechanisms may be involved in cognitive dysfunction and underlying neurobiology.

The present study has limitations. We used measures of cognition and CSF measures of $\mathrm{AD}$ in a crosssectional analysis, and the temporality of the associations cannot be extrapolated. Nevertheless, we present the most comprehensive mapping of the one-carbon pathway in both CSF and plasma in older adults. The results are encouraging and in the future should be replicated in an independent cohort as well as confirmed in relation to longitudinal change in cognition and the incidence of AD.

\section{Conclusions}

Our results suggest that several one-carbon metabolites beyond Hcy are relevant to AD pathology and cognitive function in older adults. This may partially explain why Hcy-lowering trials have been mostly disappointing and opens the possibility that other metabolites in the pathway may also be relevant targets for the prevention of cognitive decline and the underlying neurobiology of $\mathrm{AD}$.

\section{Additional file}

\begin{abstract}
Additional file 1: Figure S1. Box plots of CSF metabolite measurements for CDR of 0 and CDR $>0$ (i.e., CDR 0.5 or 1). Level of significance is indicated as $p$ value after Bonferroni correction. Values were logtransformed. Figure S2. Box plots of plasma metabolite measurements for CDR 0 and CDR $>0$ (i.e., CDR 0.5 or 1). Level of significance is indicated as $p$ value after Bonferroni correction. Concentration values were log-transformed. Figure S3. CSF and plasma metabolites correlated with CSF p-tau181 (a) and CSF tau (b) in APOE \&4 carriers $(n=37)$. Concentration values were log-transformed. Each dot represents a subject. The black line represents the linear fit, and the gray shading represents its confidence interval. Figure S4. Box plots of plasma metabolite measurements in APOE $\varepsilon 4$ carriers $(n=37)$ according to CSF p-tau181/A $\beta_{1-42}$ ratio (i.e., "low" when p-tau181/A $\beta_{1-42}$ is $\leq 0.0779$ and "high" when $p$-tau181/A $\beta_{1-42}$ is $>0.0779$ ) for negative and positive CSF profiles of AD pathology, respectively. Concentration values were $\log _{10}$-transformed. Level of significance is indicated as $p$ value after Bonferroni correction. Supplementary Methods. (PDF $831 \mathrm{~kb}$ )
\end{abstract}

\section{Abbreviations}

A $\beta$ : $\beta$-Amyloid; AD: Alzheimer disease; ANOVA: Analysis of variance; APOE: Apolipoprotein E; CDR: Clinical Dementia Rating; CSF: Cerebrospinal fluid; Hcy: Homocysteine; LASSO: Least absolute shrinkage and selection operator; LC-MS/MS: Liquid chromatography-tandem mass spectrometry; MCl: Mild cognitive impairment; MMSE: Mini Mental State Examination; 5MTHF: 5-Methyltetrahydrofolate; p-tau181: Tau phosphorylated at threonine 181; SAH: S-adenosyl-L-homocysteine; SAM: S-adenosylmethionine

\section{Funding}

This study was supported by grants from the Swiss National Research Foundation (SNF 320030_141179; to JP) and funding from the Nestlé Institute of Health Sciences.

\section{Availability of data and materials}

The datasets used and/or analyzed during the present study are available from the corresponding author upon request.

\section{Authors' contributions}

LD was responsible for study conception and design, interpretation of the analysis, and writing of the manuscript. SPG was responsible for acquisition of data and critical revision of the manuscript. JC was responsible for acquisition of data. LDS was responsible for acquisition of data. EM was responsible for the statistical analysis plan, interpretation of the analysis, and critical revision of the manuscript. DT was responsible for acquisition of data. AO was responsible for acquisition of data and critical revision of the manuscript. BM was responsible for acquisition of data. $\mathrm{HH}$ was responsible for supervision of data acquisition and critical revision of the manuscript. SM was responsible for acquisition of data. JM was responsible for acquisition of data and critical revision of the manuscript. PD was responsible for supervision of data acquisition. SC was responsible for supervision of data acquisition. FPJM was responsible for supervision of data acquisition and critical revision of the manuscript. IM was responsible for acquisition of data and critical revision of the manuscript. MK was responsible for critical revision of the manuscript. JW was responsible for study conception and design, the statistical analysis plan, the statistical analysis, interpretation of the analysis, and drafting of the "Statistical analyses" section. GLB was responsible for study conception and design, the statistical analysis plan, interpretation of the analysis, critical revision of the manuscript, and overall study supervision. JP was responsible for study conception and design, interpretation of the analysis, critical revision of the manuscript, and overall study supervision. All authors read and approved the final manuscript. 


\section{Competing interests}

LD, SPG, JC, LDS, EM, SM, JM, PD, SC, FPJM, IM, and MK are employees of Nestlé Institute of Health Sciences S.A. JW is an employee and shareholder of Quartz Bio S.A. and received consultation honoraria from Nestlé Institute of Health Sciences. GLB is an employee of Nestlé Institute of Health Sciences S.A.; an unpaid scientific advisor of the Horizon 2020 European Union-funded PROPAG-AGEING project, whose aim is to identify new molecular signatures for early diagnosis of Parkinson's disease; and an editorial board member of the Journal of Alzheimer's Disease (2009-present); and also receives research support related to cognitive decline from the National Institute on Aging, National Institutes of Health. JP received consultation honoraria from Nestle Institute of Health Sciences. DT, AO, BM, and $\mathrm{HH}$ declare that they have no competing interests.

\section{Ethics approval and consent to participate}

The institutional ethics committee of the University Hospitals of Lausanne approved the clinical protocol (number 171/2013), and all participants or their legally authorized representatives signed written informed consent forms.

\section{Publisher's Note}

Springer Nature remains neutral with regard to jurisdictional claims in published maps and institutional affiliations.

\section{Author details \\ ${ }^{1}$ Nestlé Institute of Health Sciences, École Polytechnique Fédérale de Lausanne (EPFL) Innovation Park, Bâtiment H, 1015 Lausanne, Switzerland. ${ }^{2}$ Old Age Psychiatry, Department of Psychiatry, Centre Hospitalier Universitaire Vaudois (CHUV), Lausanne, Switzerland. ${ }^{3}$ Department of Laboratories, Centre Hospitalier Universitaire Vaudois (CHUV), Lausanne, Switzerland. ${ }^{4}$ Quartz Bio, Geneva, Switzerland. ${ }^{5}$ Present address: Liggins Institute, University of Auckland, Auckland, New Zealand.}

\section{Received: 9 February 2017 Accepted: 26 May 2017}

Published online: 17 June 2017

\section{References}

1. Shane B. Folate and vitamin $B_{12}$ metabolism: overview and interaction with riboflavin, vitamin $B_{6}$, and polymorphisms. Food Nutr Bull. 2008;29(2 Suppl):S5-19.

2. Kluijtmans LAJ, Young IS, Boreham CA, Murray L, McMaster D, McNulty H, et al. Genetic and nutritional factors contributing to hyperhomocysteinemia in young adults. Blood. 2003;101:2483-8.

3. Kennedy DO. B vitamins and the brain: Mechanisms, dose and efficacy-a review. Nutrients. 2016;8:68.

4. McCully KS. Homocysteine and the pathogenesis of atherosclerosis. Expert Rev Clin Pharmacol. 2015;8:211-9.

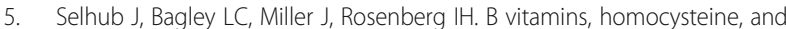
neurocognitive function in the elderly. Am J Clin Nutr. 2000;71:614S-20S.

6. Nilsson K, Gustafson L, Hultberg B. The plasma homocysteine concentration is better than that of serum methylmalonic acid as a marker for sociopsychological performance in a psychogeriatric population. Clin Chem. 2000;46:691-6.

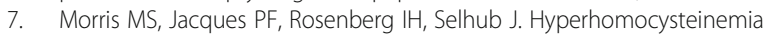
associated with poor recall in the third national health and nutrition examination survey. Am J Clin Nutr. 2001;73:927-33.

8. Lehmann M, Gottfries CG, Regland B. Identification of cognitive impairment in the elderly: homocysteine is an early marker. Dement Geriatr Cogn Disord. 1999;10:12-20.

9. Ravaglia G, Forti P, Maioli F, Martelli M, Servadei L, Brunetti N, et al. Homocysteine and folate as risk factors for dementia and Alzheimer disease. Am J Clin Nutr. 2005;82:636-43.

10. Seshadri S, Beiser A, Selhub J, Jacques PF, Rosenberg IH, D'Agostino RB, et al. Plasma homocysteine as a risk factor for dementia and Alzheimer's disease. N Engl J Med. 2002;346:476-83.

11. Faverzani JL, Hammerschmidt TG, Sitta A, Deon M, Wajner M, Vargas CR. Oxidative stress in homocystinuria due to cystathionine $\beta$-synthase deficiency: findings in patients and in animal models. Cell Mol Neurobiol. doi:10.1007/s10571-017-0478-0.

12. Vanzin CS, Mescka CP, Donida B, Hammerschimidt TG, Ribas GS, Kolling J, et al. Lipid, oxidative and inflammatory profile and alterations in the enzymes paraoxonase and butyrylcholinesterase in plasma of patients with homocystinuria due CBS deficiency: the vitamin $B_{12}$ and folic acid importance. Cell Mol Neurobiol. 2015;35:899-911.
13. Aisen PS, Schneider LS, Sano M, Diaz-Arrastia R, Van Dyck CH, Weiner MF, et al. High-dose B vitamin supplementation and cognitive decline in Alzheimer disease: a randomized controlled trial. JAMA. 2008;300:1774-83.

14. Popp J, Lewczuk P, Linnebank M, Cvetanovska G, Smulders Y, Kölsch H, et al. Homocysteine metabolism and cerebrospinal fluid markers for Alzheimer's disease. J Alzheimers Dis. 2009;18:819-28.

15. Selley ML. A metabolic link between S-adenosylhomocysteine and polyunsaturated fatty acid metabolism in Alzheimer's disease. Neurobiol Aging. 2007;28:1834-9.

16. Su SC, Tsai LH. DNA methylation in cognition comes of age. Nat Neurosci. 2012;15:1061-2

17. Vance DE, Walkey CJ, Cui Z. Phosphatidylethanolamine N-methyltransferase from liver. Biochim Biophys Acta Lipids Lipid Metab. 1997;1348:142-50.

18. Watkins SM, Zhu X, Zeisel SH. Phosphatidylethanolamine-N-methyltransferase activity and dietary choline regulate liver-plasma lipid flux and essential fatty acid metabolism in mice. J Nutr. 2003;133:3386-91.

19. Corder EH, Saunders AM, Strittmatter WJ, Schmechel DE, Gaskell PC, Small GW, et al. Gene dose of apolipoprotein E type 4 allele and the risk of Alzheimer's disease in late onset families. Science. 1993;261:921-3.

20. Kivipelto M, Rovio S, Ngandu T, Kåreholt I, Eskelinen M, Winblad B, et al. Apolipoprotein e $\varepsilon 4$ magnifies lifestyle risks for dementia: a populationbased study. J Cell Mol Med. 2008;12:2762-71.

21. Feng L, Li J, Yap KB, Kua EH, Ng TP. Vitamin B-12, apolipoprotein E genotype, and cognitive performance in community-living older adults: Evidence of a gene-micronutrient interaction. Am J Clin Nutr. 2009;89:1263-8.

22. Lee $\mathrm{YM}, \mathrm{Ha}$ JK, Park JM, Lee BD, Moon E, Chung Yl, et al. Apolipoprotein E genotype modulates effects of vitamin $B_{12}$ and homocysteine on grey matter volume in Alzheimer's disease. Psychogeriatrics. 2016;16:3-11.

23. Guiraud SP, Montoliu I, Da Silva L, Dayon L, Núñez Galindo A, Corthésy J, et al. High-throughput and simultaneous quantitative analysis of homocysteine-methionine cycle metabolites and co-factors in blood plasma and cerebrospinal fluid by isotope dilution LC-MS/MS. Anal Bioanal Chem. 2017;409:295-305.

24. Popp J, Riad M, Freymann K, Jessen F. Diagnostic lumbar puncture performed in the outpatient setting of a memory clinic: frequency and risk factors of post-lumbar puncture headache [in German]. Nervenarzt. 2007;78: 547-51.

25. Winblad B, Palmer K, Kivipelto M, Jelic V, Fratiglioni L, Wahlund LO, et al. Mild cognitive impairment - beyond controversies, towards a consensus: report of the International Working Group on Mild Cognitive Impairment. J Intern Med. 2004;256:240-6.

26. Buschke H, Sliwinski MJ, Kuslansky G, Lipton RB. Diagnosis of early dementia by the Double Memory Test: encoding specificity improves diagnostic sensitivity and specificity. Neurology. 1997;48:989-97.

27. Morris JC. The Clinical Dementia Rating (CDR): current version and scoring rules. Neurology. 1993:43:2412-4.

28. McKhann GM, Knopman DS, Chertkow H, Hyman BT, Jack Jr CR, Kawas CH, et al. The diagnosis of dementia due to Alzheimer's disease: recommendations from the National Institute on Aging-Alzheimer's Association workgroups on diagnostic guidelines for Alzheimer's disease. Alzheimers Dement. 2011;7:263-9.

29. American Psychiatric Association. Diagnostic and statistical manual of mental disorders. 4th ed. Washington, DC: American Psychiatric Association; 1994.

30. Youden WJ. Index for rating diagnostic tests. Cancer. 1950;3:32-5.

31. Tautvydaitė D, Kukreja D, Antonietti JP, Henry H, von Gunten A, Popp J. Interaction between personality traits and cerebrospinal fluid biomarkers of Alzheimer's disease pathology modulates cognitive performance. Alzheimers Res Ther. 2017;9:6.

32. Duits FH, Teunissen CE, Bouwman FH, Visser PJ, Mattsson N, Zetterberg H, et al. The cerebrospinal fluid "Alzheimer profile": easily said, but what does it mean? Alzheimers Dement. 2014;10:713-23.

33. Tibshirani R. Regression shrinkage and selection via the lasso. J R Stat Soc B. 1996;58:267-88.

34. Friedman J, Hastie T, Tibshirani R. Regularization paths for generalized linear models via coordinate descent. J Stat Softw. 2010;33:1-22.

35. Robin X, Turck N, Hainard A, Tiberti N, Lisacek F, Sanchez JC, Müller M. pROC: an open-source package for R and $\mathrm{S}+$ to analyze and compare ROC curves. BMC Bioinf. 2011;12:77.

36. DeLong ER, DeLong DM, Clarke-Pearson DL. Comparing the areas under two or more correlated receiver operating characteristic curves: a nonparametric approach. Biometrics. 1988;44:837-45. 
37. Gauher S. Is your classification model making lucky guesses? Revolutions. 22 Mar 2016. http://blog.revolutionanalytics.com/2016/03/classification-models. html. Accessed 1 Feb 2017.

38. Gardner LA, Desiderio DM, Groover CJ, Hartzes A, Yates CR, Zucker-Levin AR, et al. LC-MS/MS identification of the one-carbon cycle metabolites in human plasma. Electrophoresis. 2013;34:1710-6.

39. Tapia-Rojas C, Lindsay CB, Montecinos-Oliva C, Arrazola MS, Retamales RM, Bunout $\mathrm{D}$, et al. Is L-methionine a trigger factor for Alzheimer's-like neurodegeneration? Changes in A oligomers, tau phosphorylation, synaptic proteins. Wnt signaling and behavioral impairment in wild-type mice. Mol Neurodegener. 2015;10:62.

40. Meck WH, Williams CL. Choline supplementation during prenatal development reduces proactive interference in spatial memory. Dev Brain Res. 1999;1 18:51-9.

41. Poly C, Massaro JM, Seshadri S, Wolf PA, Cho E, Krall E, et al. The relation of dietary choline to cognitive performance and white-matter hyperintensity in the Framingham Offspring Cohort. Am J Clin Nutr. 2011;94:1584-91.

42. Breckenridge WC, Gombos G, Morgan IG. The lipid composition of adult rat brain synaptosomal plasma membranes. Biochim Biophys Acta Biomembr. 1972;266:695-707.

43. Chang PY, Lu SC, Chen CH. S-adenosylhomocysteine: a better marker of the development of Alzheimer's disease than homocysteine? J Alzheimers Dis. 2010;21:65-6.

44. Sontag E, Nunbhakdi-Craig V, Sontag JM, Diaz-Arrastia R, Ogris E, Dayal S, et al. Protein phosphatase 2A methyltransferase links homocysteine metabolism with tau and amyloid precursor protein regulation. J Neurosci. 2007:27:2751-9.

45. Obeid R, Kasoha M, Knapp JP, Kostopoulos P, Becker G, Fassbender K, et al. Folate and methylation status in relation to phosphorylated tau protein ${ }_{(181 \mathrm{P})}$ and $\beta$-amyloid(1-42) in cerebrospinal fluid. Clin Chem. 2007;53:1129-36.

46. Fuso A, Nicolia V, Cavallaro RA, Ricceri L, D'Anselmi F, Coluccia P, et al. Bvitamin deprivation induces hyperhomocysteinemia and brain $\mathrm{S}$ adenosylhomocysteine, depletes brain S-adenosylmethionine, and enhances PS1 and BACE expression and amyloid- $\beta$ deposition in mice. Mol Cell Neurosci. 2008:37:731-46.

47. Fuso A, Seminara L, Cavallaro RA, D'Anselmi F, Scarpa S. Sadenosylmethionine/homocysteine cycle alterations modify DNA methylation status with consequent deregulation of PS1 and BACE and $\beta-$ amyloid production. Mol Cell Neurosci. 2005;28:195-204.

48. Oikonomidi A, Lewczuk P, Kornhuber J, Smulders Y, Linnebank M, Semmler A, et al. Homocysteine metabolism is associated with cerebrospinal fluid levels of soluble amyloid precursor protein and amyloid beta. J Neurochem. 2016;139: 324-32.

49. Zheng L, Kong $X$, Cui $Y$, Wei $Y$, Zhang J, Wei W. Conversion from MCI to AD in patients with the APOE $\varepsilon 4$ genotype: prediction by plasma $\mathrm{HCY}$ and serum BDNF. Neurosci Lett. 2016;626:19-24.

50. Linnebank M, Popp J, Smulders Y, Smith D, Semmler A, Farkas M, et al. $S$-adenosylmethionine is decreased in the cerebrospinal fluid of patients with Alzheimer's disease. Neurodegener Dis. 2010;7:373-8.

51. Troesch B, Weber P, Mohajeri MH. Potential links between impaired onecarbon metabolism due to polymorphisms, inadequate B-vitamin status, and the development of Alzheimer's disease. Nutrients. 2016;8:63.

\section{Submit your next manuscript to BioMed Central and we will help you at every step:}

- We accept pre-submission inquiries

- Our selector tool helps you to find the most relevant journal

- We provide round the clock customer support

- Convenient online submission

- Thorough peer review

- Inclusion in PubMed and all major indexing services

- Maximum visibility for your research

Submit your manuscript at www.biomedcentral.com/submit

) Biomed Central 